\title{
Effects of Traditional Chinese Medicine Combined with Chemotherapy for Extensive-stage Small-cell Lung Cancer Patients on Improving Oncologic Survival: Study Protocol of a Multicenter, Randomized, Single- blind, Placebo-controlled Trial
}

\section{Yuyi Chen}

Department of Oncology,Beijing Hospital of Traditional Chinese Medicine,Capital Medical University,Beijing,China

\section{Mingwei Yu}

Department of Oncology,Beijing Hospital of Traditional Chinese Medicine,Capital Medical University,Beijing,China

\section{Zishen Liu}

Department of Oncology,Beijing Hospital of Traditional Chinese Medicine,Capital Medical University,Beijing,China

\section{Yi Zhang}

Department of Oncology,Beijing Hospital of Traditional Chinese Medicine,Capital Medical University,Beijing,China

\section{Qiwei Li}

Department of Oncology,Beijing Hospital of Traditional Chinese Medicine,Capital Medical University,Beijing,China

\section{GuoWang Yang ( $\sim$ guowang_yang@163.com )}

Beijing Hospital of Traditional Chinese Medine affiliated with Capital Medical University https://orcid.org/0000-0002-0799-3864

\section{Study protocol}

Keywords: Extensive-stage small-cell lung cancer (ES-SCLC), traditional Chinese medicine (TCM), randomized controlled trial (RCT), overall survival (OS), quality of life

Posted Date: January 12th, 2021

DOI: https://doi.org/10.21203/rs.3.rs-62885/v1

License: (c) (i) This work is licensed under a Creative Commons Attribution 4.0 International License. Read Full License 
Version of Record: A version of this preprint was published at Trials on July 8th, 2021. See the published version at https://doi.org/10.1186/s13063-021-05407-1. 


\section{Abstract}

Background: Extensive-stage small-cell lung cancer (ES-SCLC) is characterized by extensive metastases, aggressive progression and poor prognosis. Chemotherapy is applied as a preferred first-line regimen for ESSCLC, but inadequate for improving its overall survival. Traditional Chinese medicine (TCM) is widely used in the clinical practice of ES-SCLC for its synergy with chemotherapy. However, there is still no substantial evidence to prove that TCM can effectively improve the long-term efficacy of ES-SCLC patients. The study intends to determine whether the TCM with chemotherapy can improve the overall survival (OS) in treating with ES-SCLC when compared with chemotherapy alone.

Method/design: A multicenter, randomized, single-blind, placebo-controlled clinical trial will be conducted to determine whether the TCM granules combined with chemotherapy can improve the OS of ES-SCLC. 270 participants will randomly receive 4-6 cycles (21days per cycle) of chemotherapy plus TCM granules or placebo. The primary outcome measures is OS. The secondary outcome measures includes progression-free survival (PFS), objective response rate (ORR), quality of life (QoL) and tumor markers. Visits will be performed at the end of each cycle during the treatment period and then every 3 months in the follow-up period until the patients' death or study completion.

Discussion: The study's result will provide a high-level evidence for TCM granules using with chemotherapy on the first-line treatment of ES-SCLC.

Trial registration: Chinese Clinical Trial Registry, No. ChiCTR1900022991, registered 6 May 2019 (prospective registration).

\section{Background}

Small-cell lung cancer (SCLC) is an aggressive and rebellious neuroendocrine carcinoma, which accounts for approximately $12-15 \%$ of lung cancer ${ }^{\square}$. More than half of the newly diagnosed SCLC patients have developed into the extensive stage (ES), with lesions beyond the boundaries of hemithorax, whose 2-year mortality exceeds $95 \%$. In the past three decades, the first-line regimen of ES-SCLC have stagnated in the platinum-based chemotherapy within 9-11 months median progress free survival (PFS), which is far from meeting the cure needs ${ }^{\square}$. Even if the chemo-periods prolonged or dosages enhanced, the limited improvement in PFS and the same overall survival (OS) would come at the expense of more severe adverse events ${ }^{\square}$, probably leading to the interruption of treatment ${ }^{\square}$. The landmark breakthrough appeared in the treatment of ES-SCLC until the IMpower 133 and CASPIAN, adding the immune-checkpoint inhibitors (ICls) to the standard etoposide and platinum ${ }^{\square}$. Although this combination also tends to a heavier treatment burden because of its overprices and immunerelated adverse reactions ${ }^{\square}$, it does inspire a strategy for combining multiple treatments to benefit more ESSCLC, especially on the grounds of chemotherapy, as some studies have tried ${ }^{\mathrm{DL}}$. Thus, it is of great value to enrich the first-line regimens of ES-SCLC if therapy with better practicability and cost-effectiveness can be found in cooperation with chemotherapy.

Through a series of meta-analysis, traditional Chinese medicine (TCM) has been shown to improve the efficacy and safety with chemotherapy in varieties of malignancies, such as breast cancer ${ }^{\square}$, colorectal cancer ${ }^{\square}$ as well 
non-small cell lung cancer ${ }^{\square}$. TCM regimens have also increasingly widely used in the clinical practice of ESSCLC for its synergy with chemotherapy. In previous researches ${ }^{\square}$, the long-term and regular TCM regimens were preliminarily considered as a favorable prognosis factor of ES-SCLC. Unfortunately, due to the small sample size and substandard design, no study so far has provided a clear-cut answer to whether TCM regimen can synergize chemotherapy to achieve longer oncologic survival of ES-SCLC. Therefore, we organized a randomized controlled trial (RCT) with relatively high-quality to verify the prognostic value of TCM on ES-SCLC.

The primary objective of this RCT is to determine whether the TCM granules with chemotherapy can improve the OS in treating with ES-SCLC when compared with chemotherapy alone. The trial will also provide clinical evidence on the efficacy of TCM granules in progression-free survival (PFS), objective response rate (ORR), quality of life (QoL) and tumor markers of ES-SCLC, alongside a safety analysis.

\section{Methods/design}

\subsection{Study design}

This is a multicenter, randomized, single-blind, placebo-controlled clinical trial. After a run-in period within 7 days, 270 eligible participants will be randomized to the test group (TCM granules with chemotherapy) or the control group (placebo with chemotherapy) in a 1:1 ratio. All participants will undergo a treatment period of 1218 weeks and a follow-up period until death. During the treatment period, both groups have to receive chemotherapy for 4-6 cycles. In the interphase of chemotherapy (days 6-21 of each cycle), participants in the test group will take TCM granules while those in the control group will take placebo granules for 15 days. Visits will be performed at days $0,21,42,63,84,105,126$ in the treatment period, and the last week of every 3 months (approximately 90 days) during the subsequent follow-up period. The study is scheduled to open from December 2018 to December 2021. The recruiting was started in June 2019 and lasted for 24 months. The endpoint of follow-up is the date of death or December 31, 2021. (Fig. 1)

\subsection{Settings and participates}

Participants will be recruited from six general hospitals located in three different provinces in China, including Beijing Hospital of Traditional Chinese Medicine, Capital Medical University, Dongfang Hospital affiliated to Beijing University of Chinese Medicine, China-Japan Friendship Hospital, Longhua Hospital affiliated to Shanghai University of Traditional Chinese, Hubei Provincial Hospital of Traditional Chinese Medicine and Beijing Hospital.

Recruitment is done by posting advertisements or disseminating notices at the website and official WeChat of the six hospitals. The inclusion/exclusion and withdrawal criteria are shown in Table 1. If participants meet the withdrawal criteria, the investigators should record the reasons on the case report form (CRF) and follow up periodically as possible until the endpoint. 
Table 1

Eligibility and withdraw criteria

\section{Inclusion criteria}

1 Small cell lung cancer with confirmed cytologically or histologically diagnosis and measurable lesions.

2 Diagnosis of Extensive-disease following the Veterans Administration Lung Study Group staging criteria.

3 Participants who haven't received surgery, radiotherapy, chemotherapy or immunotherapy before.

4 ECOG PS 0-2, and expected survival over 3 months.

$5 \quad$ Aged $18-75$ years old.

6 Agree to sign of the informed consent voluntarily .

\section{Exclusion criteria}

1 Participants with dyspnea due to massive pleural, ascites, or pericardial effusion.

2 Participants have symptomatic bone metastases and need to radiotherapy.

3 Brain metastasis.

4 Presence of pregnancy, lactation, and severe primary diseased in the heart, liver, kidney or hematological system.

5 Allergy to drugs used in this study.

6 Participants undergoing other clinical trials.

\section{Withdrawal criteria}

1 Antineoplastic proprietary Chinese medicine, TCM decoction or injection outside of the protocol was used during the treatment period.

2 Participants intend to receive radiotherapy, immunotherapy and other antineoplastic therapies during the treatment period.

3 Participangts are no longer suitable to remain in the trial because of their complications.

4 Severe adverse events.

5 Participants' compliance are poor enough to affect the evaluation of efficacy and safety.

6 Participants ask to withdraw from the trial for any reason.

7 Ineligible participants who have registered by mistake must withdraw from the trial in time.

8 The study is prematurely terminated or suspended for safety concerns.

9 Participants lost to follow-up.

Abbreviations: ECOG: Eastern Cooperative Oncology Group, PS: performance status. TCM: traditional Chinese medicine.

\subsection{Intervention}

The protocol interventions consist of chemotherapy and TCM regimens. 


\subsubsection{Chemotherapy}

All the eligible participants will undergo 4-6 cycles (21days per cycle) chemotherapy with the standard regimens recommended by the National Comprehensive Cancer Network (NCCN) clinical practice guidelines in SCLC (Version 2, 2018), and the preferred one is EP (Etoposide $100 \mathrm{mg} / \mathrm{m}^{2}$ on day $1-3$, Cisplatin $75 \mathrm{mg} / \mathrm{m}^{2}$ on day 1 , every 21 days).

\subsubsection{TCM regimens}

The TCM regimens including six kinds of granules corresponding to the six most common types of TCM syndromes in ES-SCLC patients. As shown in Table 2, participants with indications of a syndrome type, including all pulmonary symptoms, tongue and pulse, and more than two systemic symptoms, will be diagnosed with the type by TCM physicians and received the matched granules. Since participants' symptoms are likely to change over time along with the treatment, the diagnosis of types will be redone before each cycle to determine which kind of granules is the most tailored one. 
Table 2

Indications of TCM granules and placebo

\begin{tabular}{|c|c|c|c|c|}
\hline \multirow{2}{*}{$\begin{array}{l}\text { Name of } \\
\text { granules }\end{array}$} & \multirow[t]{2}{*}{ Syndrome types } & \multicolumn{3}{|l|}{ Indications } \\
\hline & & $\begin{array}{l}\text { Pulmonary } \\
\text { symptoms }\end{array}$ & Systemic symptoms & Tongue and pulse \\
\hline $\begin{array}{l}\text { TCM } \\
\text { Recipe } 1 \\
\text { Placebo } \\
\text { Recipe } 1\end{array}$ & $\begin{array}{l}\text { Qi-Ying } \\
\text { deficiency } \\
\text { syndrome }\end{array}$ & $\begin{array}{l}\text { Cough weakly } \\
\text { with little } \\
\text { phlegm. }\end{array}$ & $\begin{array}{l}\text { Fatigue, sweating, hot } \\
\text { flashes, palpitations. }\end{array}$ & $\begin{array}{l}\text { Reddish tongue with thin } \\
\text { or less coating, thready } \\
\text { pulse. }\end{array}$ \\
\hline $\begin{array}{l}\text { TCM } \\
\text { Recipe } 2 \\
\text { Placebo } \\
\text { Recipe } 2\end{array}$ & $\begin{array}{l}\text { Lung-spleen Qi } \\
\text { deficiency } \\
\text { syndrome }\end{array}$ & $\begin{array}{l}\text { Cough and } \\
\text { asthma with } \\
\text { white dilute } \\
\text { sputum. }\end{array}$ & $\begin{array}{l}\text { oppression in chest, } \\
\text { fatigue, loss of appetite, } \\
\text { abdominal distension, } \\
\text { dropsy, loose stools. }\end{array}$ & $\begin{array}{l}\text { Plump tongue with tooth } \\
\text { prints and white greasy } \\
\text { coating, sunken and } \\
\text { thready pulse. }\end{array}$ \\
\hline $\begin{array}{l}\text { TCM } \\
\text { Recipe } 3 \\
\text { Placebo } \\
\text { Recipe } 3\end{array}$ & $\begin{array}{l}\text { Lung-Yin } \\
\text { deficiency } \\
\text { syndrome }\end{array}$ & $\begin{array}{l}\text { Dry cough } \\
\text { without } \\
\text { phlegm. }\end{array}$ & $\begin{array}{l}\text { Hot flashes, sweating, } \\
\text { thirsty, irritability, hoarse } \\
\text { voice. }\end{array}$ & $\begin{array}{l}\text { Red tongue with less } \\
\text { coating, quick and } \\
\text { thready pulse. }\end{array}$ \\
\hline $\begin{array}{l}\text { TCM } \\
\text { Recipe } 4 \\
\text { Placebo } \\
\text { Recipe } 4\end{array}$ & $\begin{array}{l}\text { Qi stagnation } \\
\text { and blood } \\
\text { stasis } \\
\text { syndrome }\end{array}$ & $\begin{array}{l}\text { Cough and } \\
\text { dyspnea with } \\
\text { phlegm difficult } \\
\text { to cough up. }\end{array}$ & $\begin{array}{l}\text { Stabbing or distending } \\
\text { pain, subcutaneous } \\
\text { haemorrhage, depression } \\
\text { and anxiety. }\end{array}$ & $\begin{array}{l}\text { Cyanotic tongue with thin } \\
\text { coating, wiry and } \\
\text { astringent pulse. }\end{array}$ \\
\hline $\begin{array}{l}\text { TCM } \\
\text { Recipe } 5 \\
\text { Placebo } \\
\text { Recipe } 5\end{array}$ & $\begin{array}{l}\text { Heat-phlegm } \\
\text { obstructing } \\
\text { lung syndrom }\end{array}$ & $\begin{array}{l}\text { Cough with } \\
\text { excessive } \\
\text { yellow sticky } \\
\text { phlegm. }\end{array}$ & $\begin{array}{l}\text { Fever, oppression and pain } \\
\text { in chest, hemoptysis, thirst. }\end{array}$ & $\begin{array}{l}\text { Red tough with yellow } \\
\text { greasy coating, quick and } \\
\text { slippery pulse. }\end{array}$ \\
\hline $\begin{array}{l}\text { TCM } \\
\text { Recipe } 6 \\
\text { Placebo } \\
\text { Recipe } 6\end{array}$ & $\begin{array}{l}\text { Qi deficiency } \\
\text { with excessive } \\
\text { cancerous toxin } \\
\text { syndrome }\end{array}$ & $\begin{array}{l}\text { Cough is } \\
\text { aggravating, } \\
\text { and lesions in } \\
\text { lung } \\
\text { progressed. }\end{array}$ & $\begin{array}{l}\text { Weight loss, fatigue, loss } \\
\text { of appetite, chest pain, } \\
\text { cancerous fever, } \\
\text { hemoptysis, sweating. }\end{array}$ & $\begin{array}{l}\text { Dark tough without luster, } \\
\text { thick greasy or denuded } \\
\text { coating, sunken and wiry } \\
\text { pulse. }\end{array}$ \\
\hline $\begin{array}{l}\text { Abbreviati } \\
\text { participan } \\
\text { systemic } \\
\text { randomiz }\end{array}$ & $\begin{array}{l}\text { s: TCM: tradition } \\
\text { as its pulmonary } \\
\text { aptoms. The cor } \\
\text { n. }\end{array}$ & $\begin{array}{l}\text { hinese medici } \\
\text { nptoms, supp } \\
\text { onding granul }\end{array}$ & $\begin{array}{l}\text { he diagnosis criteria of a } \\
\text { evidence of tongue and } \\
\text { II be used in accordance }\end{array}$ & $\begin{array}{l}\text { drome type is that the } \\
\text { e, and more than two } \\
\text { syndrome types and }\end{array}$ \\
\hline
\end{tabular}

Participants in the test group will take TCM granules with $200 \mathrm{ml}$ warm water twice daily for 15 days from the sixth day of each chemotherapy cycle. The herbs and dosages of the TCM granules are shown in Supplement 1. While those in the control group will take placebo granules composed of $95 \%$ dextrin and 5\% TCM granules of each type in the same way.

Both the TCM granules and placebo are made uniformly in Beijing Tcmages Pharmaceutical Co. LTD (Shunyi District, Beijing, Datong Road, No. 103, 10098) to ensure the similarity in appearance, smell, texture and taste.

\subsection{Randomization and allocation concealment}


Centralized randomization was carried out by Peking University clinical research institute (PUCRI) with using the REDCap system (Version 8.4.3), a remote electronic data capture (EDC). When an eligible participant is enrolled, the clinical research coordinator (CRC) has to register the detailed information of the participant on the REDCap website. Then, after the confirmation of the principal investigator (PI) or sub-investigator (Sub-I), the participant will be assigned to either the test or control groups in an allocation ratio of 1:1. The REDCap system will automatically generate the participant's ID numbers and feedback the grouping results to PI or Sub-I, who will next inform the drug administrator to prepare TCM granules or placebo granules for the participant.

Single-blind is used in the study (Fig. 2). After being informed of grouping and syndrome type, the drug administrator has to select the correct drug from storage, where the TCM granules are packaged in red boxes while the placebos in green. Then, he will remove the granules' sachets from the red/green box to a white box and label the original red/green box and the current white box respectively with the same code that hides the information of the participant's group and type. While keeping the red/green box as evidence of single-blind, the drug administrator needs to give the labeled white box to the investigator and finally pass them to the participant. The participants and statistical analysts are blinded until the trial is completed.

\subsection{Sample size calculation}

The sample size was calculated by PASS 15.0 software. The model was established according to the log-rank test of survival analysis (bilateral side), with $a=0.05$ and power $1-\beta=0.8$. Based on recent researches ${ }^{[15][}$, the median survival in ES-SCLC with first-line chemotherapy was approximately 8 months, which we estimated to be 4 months longer after TCM combined with chemotherapy. The recruitment period was set as 24 months while the total time was 36 months. The minimum sample size was calculated as 112 in each group. Considering a 10\% loss, we sought to enroll at least 270 (135 per group) participants in the study.

\subsection{Assessments and time-points}

Assessments will be performed at baseline and every protocol-defined intervals (Table 3) until the occurrence of death or study completion. 
Table 3

Schedule of data collection

\begin{tabular}{|c|c|c|c|c|c|c|c|c|c|c|c|}
\hline \multirow[b]{2}{*}{ Visits } & \multirow{2}{*}{$\begin{array}{l}\text { Screening\&baseline } \\
\text { - }\end{array}$} & \multicolumn{6}{|c|}{ Treatment period } & \multicolumn{4}{|c|}{ Follow-up period } \\
\hline & & 1 & 2 & 3 & 4 & 5 & 6 & 7 & 8 & 9 & ....... \\
\hline $\begin{array}{l}\text { Time points } \\
\text { (days) }^{a}\end{array}$ & -7 to 0 & 21 & 42 & 63 & 84 & 105 & 126 & 195 & 285 & 375 & ....... \\
\hline $\begin{array}{l}\text { Time windows } \\
\text { (days) }\end{array}$ & - & $\frac{ \pm}{7}$ & $\frac{ \pm}{7}$ & $\frac{ \pm}{7}$ & $\frac{ \pm}{7}$ & \pm 7 & \pm 7 & $\stackrel{ \pm}{21}$ & $\frac{ \pm}{21}$ & $\stackrel{ \pm}{21}$ & $\stackrel{ \pm}{21}$ \\
\hline Informed consent & $x$ & & & & & & & & & & \\
\hline $\begin{array}{l}\text { Inclusion and } \\
\text { exclusion criteria }\end{array}$ & $x$ & & & & & & & & & & \\
\hline Survival state & & $x$ & $x$ & $x$ & $x$ & $x$ & $x$ & $x$ & $x$ & $x$ & $x$ \\
\hline General data & $x$ & & & & & & & & & & \\
\hline $\begin{array}{l}\text { History of } \\
\text { diagnosis and } \\
\text { treatment }\end{array}$ & $x$ & & & & & & & & & & \\
\hline ECOG-PS & $x$ & & & & & & & & & & \\
\hline $\begin{array}{l}\text { Imaging } \\
\text { examination }\end{array}$ & $x$ & & $x$ & & $x$ & & $x$ & $x$ & $x$ & $x$ & $x$ \\
\hline ORR & & & $x$ & & $x$ & & $x$ & $x$ & $x$ & $x$ & $x$ \\
\hline FACT-L scale & $x$ & $x$ & $x$ & $x$ & $x$ & $x$ & $x$ & $x$ & $x$ & $x$ & $x$ \\
\hline $\begin{array}{l}\text { TCM symptoms } \\
\text { scale }\end{array}$ & $x$ & $x$ & $x$ & $x$ & $x$ & $x$ & $x$ & $x$ & $x$ & $x$ & $x$ \\
\hline Tumor markers & $x$ & $x$ & $x$ & $x$ & $x$ & $x$ & $x$ & & & & \\
\hline $\begin{array}{l}\text { Blood, urine, stool } \\
\text { routine } \\
\text { examination }\end{array}$ & $x$ & $x$ & $x$ & $x$ & $x$ & $x$ & $x$ & & & & \\
\hline $\begin{array}{l}\text { Liver and renal } \\
\text { function } \\
\text { examination }\end{array}$ & $x$ & $x$ & $x$ & $x$ & $x$ & $x$ & $x$ & & & & \\
\hline Electrocardiogram & $x$ & $x$ & $x$ & $x$ & $x$ & $x$ & $x$ & & & & \\
\hline AEs & & $x$ & $x$ & $x$ & $x$ & $x$ & $x$ & $x$ & $x$ & $x$ & $x$ \\
\hline $\begin{array}{l}\text { Compliance } \\
\text { assessment }\end{array}$ & & $x$ & $x$ & $x$ & $x$ & $x$ & $x$ & $x$ & $x$ & $x$ & $x$ \\
\hline
\end{tabular}

Abbreviations: ECOG-PS: Eastern Cooperative Oncology Group performance status, ORR: objective response rate, FACT-L: Functional Assessment of Cancer Therapy-lung carcinoma,TCM: traditional Chinese medicine, AEs: adverse events.

${ }^{a}$ At the end of each cycle in the treatment period and every three months in the follow-up period. 


\section{Screening\&baseline Treatment period}

Follow-up period

Drug combination

$x$

$x$

$x$

$x$

$x$

$x$

$x$

$\times$

$x$

$x$

Abbreviations: ECOG-PS: Eastern Cooperative Oncology Group performance status, ORR: objective response rate, FACT-L: Functional Assessment of Cancer Therapy-lung carcinoma,TCM: traditional Chinese medicine, AEs: adverse events.

${ }^{a} A t$ the end of each cycle in the treatment period and every three months in the follow-up period.

\subsubsection{Primary outcome}

The primary outcome is overall survival (OS), from randomization to death.

\subsubsection{Secondary outcomes}

The secondary outcomes are as follow:

PFS: from randomization to occurrence of imaging-based disease progress (PD), which is assessed by the investigators.

ORR: Imaging examinations for the target lesions will be done at days $0,42,84,126$ during the treatment period and every 90 days during the follow-up period. The tumor response is evaluated according to standards of Response Evaluation Criteria in Solid Tumors (RECIST) Version 1.1, rating as complete response (CR), partial response (PR), stable disease (SD) or PD. ORR is the ratio of participants rated as CR and PR to total cases.

QoL: Functional Assessment of Cancer Therapy-lung carcinoma (FACT-L) scale and TCM syndromes scale are used for the evaluation of QoL. The participants will answer the questionnaires in FACT-L independently with the assistance of investigators, covering physical, social\&family, emotional and functional statuses, as well as lung cancer module. The total score of FACT-L is calculated by adding up the scores of each item. TCM syndrome scale is designed to record the extent of several typical symptoms such as cough, phlegm, fatigue, shortness of breath and loss of appetite. 0, 1, 2, 3 points represent "none", "mild", "moderate", "severe", respectively. QoL data are collected at baseline and in each visit.

Tumor markers: the commonest tumor markers, NSE and proGRP, are tested at days $0,21,42,63,84,105,126$ during the treatment period.

\subsubsection{Safety assessment}

The participants' blood, urine and stool routine, liver and kidney function, electrocardiogram were monitored at days $0,21,42,63,84,105,126$ during the treatment period. The AEs are categorized according to the National Cancer Institute's Common Terminology Criteria for Adverse Events (NCI-CTCAE) Version 4.03 and recorded overall the study.

\subsection{Register, ethical issues and oversight}

Before recruitment, we have registered the protocol in the Chinese Clinical Trial Registry (No.

ChiCTR1900022991, 6 May 2019). 
The study was ethically approved (2019L02-009-01) by the Research Ethical Committees at Beijing Hospital of the Traditional Chinese Medicine, Capital Medical University and the other five participating sites. Now it is being conducted according to the principles of risks and benefits, privacy and confidentiality, informed consent, placebo using, etc. in the declaration of Helsinki.

The oversight is in charge of Qihuang Contract Research Organization (CRO), through quarterly monitoring by clinical research associates (CRA) in terms of safety, quality and progress of the study.

\subsection{Data management}

PUCRI is responsible for data management. The data managers (DM) in PUCRI have built an EDC on the basis of CRF in REDCap system (Version 8.4.3) according to "data management and verification plan" drafted before. CRCs can type-in data to the EDC through their personal accounts. The errors or questions discovered by the system will be sent to the CRCs for answers. After all the data inputted without remaining questions, PI, sub-Is and CRCs will lock the database within 4 weeks. Finally, the database will be exported by PUCRI and hand over to the statisticians.

\subsection{Statistical analysis}

All data will be processed by a two-sided statistical test in SAS software 9.2 (the installation site's authorization number: 11202165). $P \leq 0.05$ is defined as a statistical significance. Full analysis set (FAS), per-protocol set (PPS) and safety set (SS) will be created after data collection.

The statistical design is as follows:

For characteristics at baseline, continuous variables will be described with the use of mean (standard deviation) or median (quartile) based on the distribution. A T-test will be adopted if the normality and homogeneity of variance are satisfied, otherwise, the nonparametric test will be carried out. Categorical variables will be analyzed by the Chi-square test or nonparametric test.

For OS and PFS, the Kaplan-Meire curve and the Log-rank test will be used to analyze the distribution of the survival function. And the factors selected in the univariate analysis will be brought into the Cox regression for further multivariate analysis.

For tumor response, the logistic regression or Ridit analysis will be used to compare the differences in ORR. While the proportion of CR, PR, SD, PD will be compared by the CHM test because of the central effect.

For QoL and tumor markers, the T-test or RMANOVA will be used to compare the differences intra- or intergroups if the two groups' scores at baseline are similar. Or the covariance analysis will be used to correct for confounders if not.

For safety assessment, the AEs and their incidences should be recorded in detail.

\section{Discussion}

To our knowledge, this is the first multicenter RCT aiming at long-term efficacy and prognosis of TCM granules with chemotherapy in ES-SCLC patients. Two published cohort research ${ }^{[15][}$ have reached an opposite 
conclusion about whether TCM decoctions can improve the PFS of ES-SCLC. Apart from the fact that both of their sample sizes were too small to be credible, they all made TCM as the only intervention but ignored the possibilities for patients choosing standard regimens simultaneously, which wasn't quite consistent with the present treatment status of ES-SCLC in China. Another RCT ${ }^{[}$of 80 SCLC cases demonstrated that TCM decoctions with standard EP could achieve a higher DCR versus EP alone. However, the study didn't explain its randomization and blind. We suspect it may be an open-label trial, for no placebo was found. More crucially, the study didn't stratify the limited and extensive stage in statistical analysis, which reduced the reference value of the results. Despite the lack of stringent design, the above studies have all affirmed the effectiveness of TCM on SCLC, especially in improving QoL. By contrast, our study does have several strengthens and innovations. First of all, this is a multicenter, randomized, single-blind, placebo-controlled clinical trial. The processes of grouping and single-blind are clear and well-documented. And the participants are from six grade-A tertiary hospitals in China, ensuring the representation of samples. The data are collected and managed on EDC, under the oversight of an independent monitoring organization, which is related to the credibility of our future findings. Secondly, our sample size is larger than previous similar studies. Thirdly, we focus on the role of TCM in prognosis, not just in reducing the toxicity or improving the QoL. The survival benefit is the key to judge TCM's necessity in the treatment of ES-SCLC. And our study will solve the mystery. In addition, the multiple prescriptions in TCM regimens were designed for the common syndrome types of ES-SCLC, so as to realize the potentials and advantages of TCM syndrome differentiation.

The study still has some limitations as follows. First, the study wasn't designed as a double-blind trial. The traditional double-blind double-mimic method is not affordable and suitable for this study of multiple TCM prescriptions. Therefore, we finally chose single-blind which conforms to the complexity and feasibility of TCM and implemented it strictly to control the risk of reveals. Second, the assessment of OS may be influenced by confounders. Since the study intents to evaluate the long-term efficacy of TCM granules with chemotherapy, the TCM intervention period is designed as 4-6 cycles, synchronizing with chemotherapy. While in recent years, the second-line or later regimens are continuously refreshed by $\mathrm{ICls}^{\square}$, anlotinib ${ }^{\square}$, lurbinectedin ${ }^{\square}$ and Rovalpituzumab Tesirine (Rova-T) ${ }^{\square}$. As the study gives priority to the patient's interests, the investigators should respect participants' choices of positive treatment after PD. Thus, the second- and third-line regimen may become vital factors for prognosis. As a remedy, we require the investigators to keep detailed records of regimen participants using after PD and provide them to statisticians to build a more realistic and reasonable regression model.

In conclusion, the study's result will provide a high-level evidence for TCM granules using with chemotherapy on the first-line treatment of ES-SCLC.

\section{Trial Status}

The trial is currently enrolling participants.

\section{Declarations}

\subsection{Ethics approval and consent to participate}


Before recruitment, we have registered the protocol in the Chinese Clinical Trial Registry (No.

ChiCTR1900022991, 6 May 2019). The study was ethically approved (2019L02-009-01) by the Research Ethical Committees at Beijing Hospital of the Traditional Chinese Medicine, Capital Medical University.

\subsection{Consent for publication}

All the authors are willing to provide a model consent form on request.

\subsection{Availability of data and materials}

Not applicable.

\subsection{Competing interests}

The authors declare that they have no competing interests.

\subsection{Funding}

The study was supported by National Key Research \& Development Program of China [grant number: 2018YFC1705101].

\subsection{Authors' contributions}

All authors participated in the design of the study. YYC wrote the draft of manuscript. GWY is responsible for coordinating the clinical trial. GWY, MWY and YYC participated in statistical design. ZSL is responsible for recruiting the participants. GWY, MWY, YZ and QWL substantively revised the manuscript. All the authors approved the submitted version.

\subsection{Acknowledgments}

First of all, we thank all the participants and their families for their participation and support in this study. We have to express our gratitude to the clinicians who contribute to the study in Beijing Hospital of Traditional Chinese Medicine, Capital Medical University, Dongfang Hospital affiliated to Beijing University of Chinese Medicine, China-Japan Friendship Hospital, Longhua Hospital affiliated to Shanghai University of Traditional Chinese and Hubei Provincial Hospital of Traditional Chinese Medicine and Beijing Hospital. We also thank Beijing Qihuang Medicine Clinical Research Center for oversight and Peking University clinical research institute for statistical advice and assistance.

\section{References}

1. Giandomenico R, Laura Z, Maria RC. No Advantage in Survival With Targeted Therapies as Maintenance in Patients With Limited and Extensive-Stage Small Cell Lung Cancer: A Literature-Based Meta-Analysis of Randomized Trials. Clinical Lung Cancer, 2016; 17(5): 334-340.

2. Filippos Koinis, Athanasios Kotsakis, Vasileios Georgoulias.Small cell lung cancer (SCLC): no treatment advances in recent years. Translational lung cancer research, 2016; 5(1):39-50. 
3. Hermes A,Bergman B,Bremnes R,et al.Irinotecan plus carboplatin versus oral etoposide plus carboplatin in extensive small-cell lung cancer: a randomized phase III trial.J Clin Oncol, 2008; 26: 4261-4267.

4. Lima JP, dos Santos LV, Sasse EC, et al. Optimal duration of first-line chemotherapy for advanced non-small cell lung cancer: a systematic review with meta-analysis. Eur J Cancer. 2009; 45(4):601-7.

5. Hang Zhou, Chao Zeng, Yang Wei, et al. Duration of chemotherapy for small cell lung cancer: a metaanalysis. PLoS One.2013; doi:10.1371/journal.pone.0073805.

6. Schiller JH, Adak S, Cella D, et al. Topotecan versus observation after cisplatin plus etoposide in extensivestage small-cell lung cancer: E7593-a phase III trial of the ECOG. J Clin Oncol. 2001 Apr 15;19(8):2114-22.

7. Samantha A. Armstrong, Stephen V. Liu. Dashing decades of defeat: long anticipated advances in the firstline treatment of extensive-stage small cell lung cancer. Current Oncology Reports. 2020; 22(2):20.

8. Mansfield AS, Każarnowicz A, Karaseva N, et al. Safety and patient-reported outcomes of atezolizumab, carboplatin, and etoposide in extensive-stage small-cell lung cancer (IMpower133): a randomized phase I/III trial.Annals of oncology. 2020; 31(2):310-317.

9. Ling-Yu Li, Hong Wang, Xiao Chen, et al.First-line atezolizumab plus chemotherapy in treatment of extensive small cell lung cancer: a cost-effectiveness analysis from China.Chinese Medical Journal.2019; 132(23):2790-2794.

10. Sibo Tian,Xinyan Zhang, Renjian Jiang,et al.Survival Outcomes with Thoracic Radiotherapy in ExtensiveStage Small Cell Lung Cancer: A Propensity-Score Matched Analysis of the National Cancer Data Base.Lung Cancer,2019.DOI: https://doi.org/10.1016/j.cllc.2019.06.014.

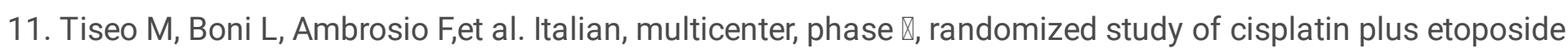
with or without bevacizumab as first-line treatment in extensive-disease small-cell lung cancer: the GOIRCAIFA FARM6PMFJM trial.J Clin Oncol.2017,35(12):1281-1287.

12. Xu Sun, Xing Zhang, Jia-Yun Nian, et al. Chinese herbal medicine as adjunctive therapy to chemotherapy for breast cancer: a systematic review and meta-analysis. Evid Based Complement Alternat Med. 2016; doi: $10.1155 / 2016 / 3281968$.

13. Shuang Lin, Xiaoxia An, Yong Guo, et al. Meta-analysis of stragalus-containing traditional Chinese medicine combined with chemotherapy for colorectal cancer: efficacy and safety to tumor response[J]. Front Oncol. 2019;9:749.

14. Zhong-liangLiu, Wei-rongZhu, Wen-chaoZhou,et al.Efficacy of traditional Chinese Medicine combined with chemotherapy in patients with non-small cell lung cancer (NSCLC): a meta-analysis of randomized clinical trials. J Integr Med. 2014; 12(4):346-58.

15. Liu R, He SL, Zhao YC, et al. Chinese herbal decoction based on syndrome differentiation as maintenance therapy in patients with extensive-stage small-cell lung cancer: an exploratory and small prospective cohort study. Evidence-Based Complementary and Alternative Medicine.2015; doi: 10.1155/2015/601067.

16. Bernhardt EB, Jalal SI.Small cell lung cancer. Cancer Treatment and Research. 2016; 170:301-322.

17. Meiping Xue, Likun Liu, Yanrong Guo, et al. Clinical study on small cell lung cancer in the maintenance treatment with Chinese herbal medicine. World Journal of Integrated Traditional and Western Medicine.2019;12(9):1271-1274.

18. Qiang Dong, Guiqin Liu, Jianxin Liu,et al. A clinical trial of Xue Fu Zhu Yu decoction in the treatment of advanced small cell lung cancer. HeNan Traditional Chinese Medicine.2014;34(9): 1758-1760. 
19. Wade T. Iams, Jason Porter, Leora Horn. Immunotherapeutic approaches for small-cell lung cancer.Nat Rev Clin Oncol. 2020;17(5):300-312.

20. Y. Cheng, Q. Wang, K. Li, et al. OA13.03 Anlotinib as third-line or further-line treatment in relapsed SCLC: a multicentre, randomized, double-blind phase 2 trial. Journal of Thoracic Oncology, 2018;13(10):S351-352.

21. JoséTrigo, VivekSubbiah, BenjaminBesse, et al. Lurbinectedin as second-line treatment for patients with small-cell lung cancer: a single-arm, open-label, phase 2 basket trial. Lancet Oncol. 2020;21(5):645-654.

22. Daniel Morgensztern, Benjamin Besse, Laurent Greillier, et al. Efficacy and safety of rovalpituzumab tesirine in third-line and beyond patients with DLL3-expressing, relapsed/refractory small-cell lung cancer: results from the phase II TRINITY study. Clin Cancer Res.2019;25(23):6958-6966.

\section{Figures}

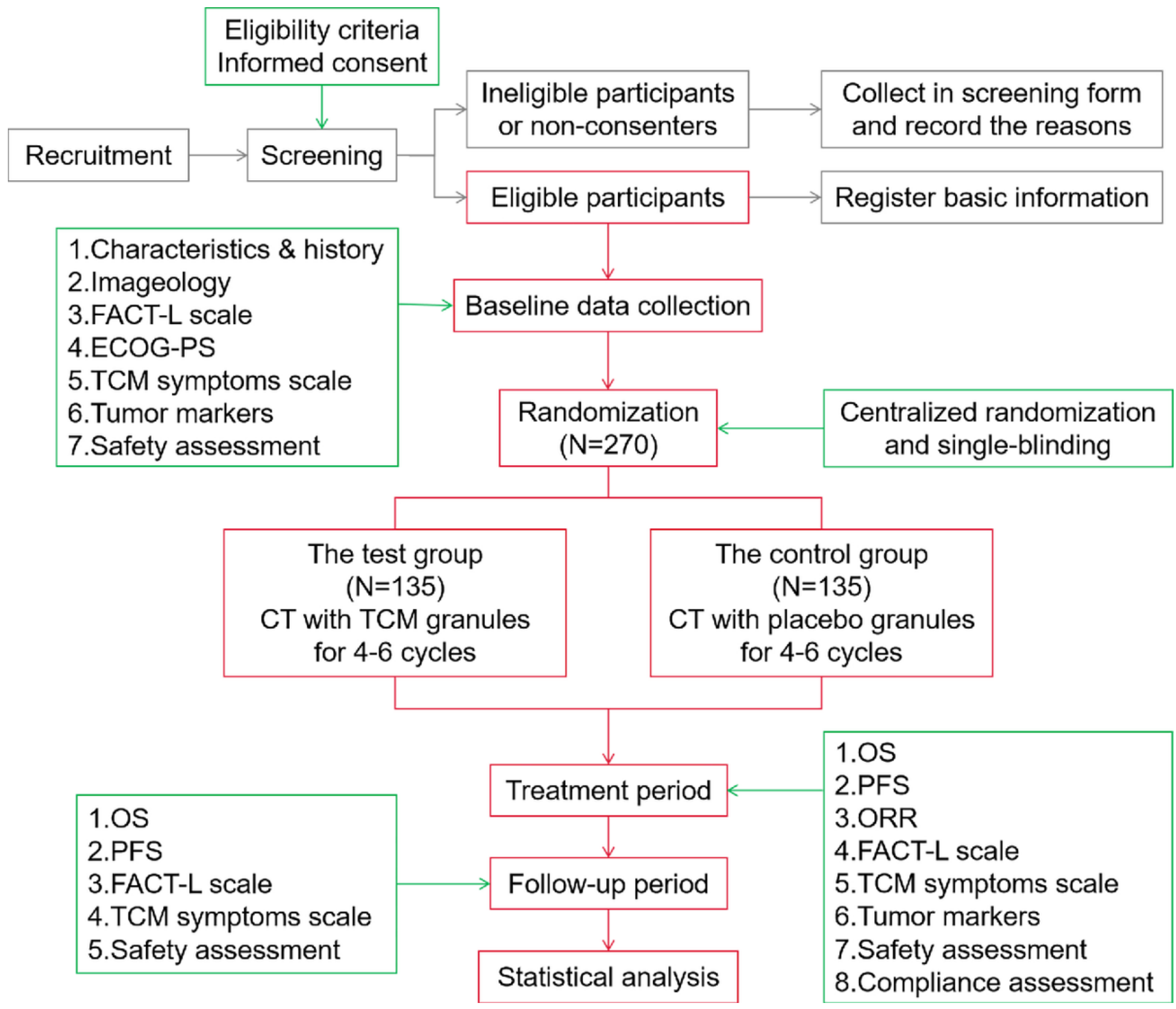

Figure 1 
Flow diagram of study design OS: overall survival, PFS: Progression-free survival, ORR: objective response rate, ECOG-PS: Eastern Cooperative Oncology Group performance status, FACT-L: Functional Assessment of Cancer Therapy-lung carcinoma, CT: chemotherapy, TCM: traditional Chinese medicine.
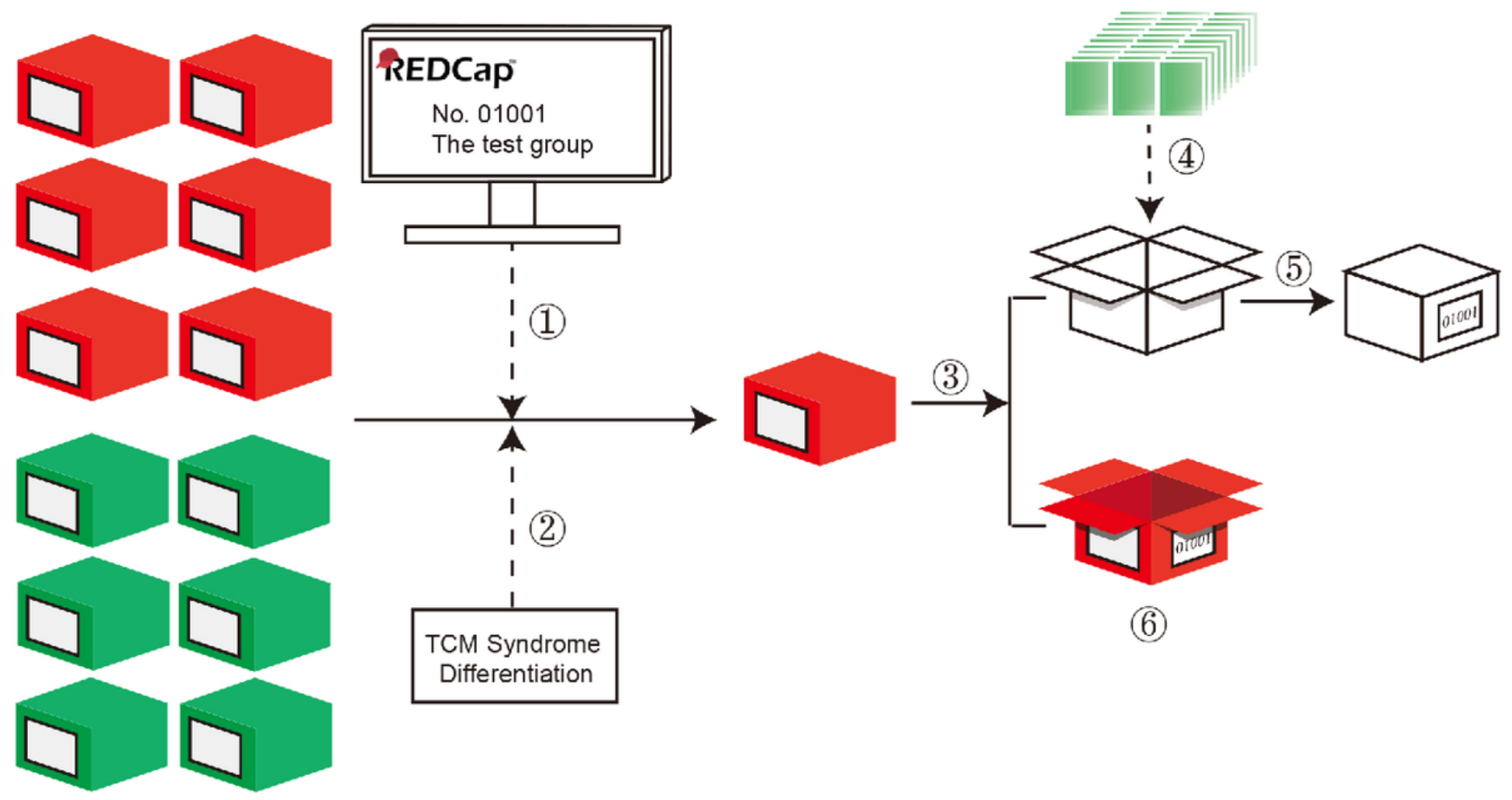

Figure 2

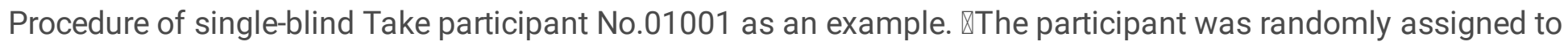
the test group by REDCap system. $\triangle$ The participant's TCM syndrome type was diagnosed by a TCM physician

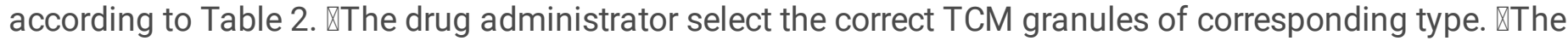
sachets of TCM granules were removed from the red box to a white box. $\triangle$ The white box was labeled by No.01001 and passed to the participant, who were blinded until the end of the study. $\otimes T h e$ red box was labeled too and kept as evidence of single-blind by the drug administrator.

\section{Supplementary Files}

This is a list of supplementary files associated with this preprint. Click to download.

- Supplement.docx 